\title{
REVIEW OF "THE GLOBALIZATION OF \\ INTERNATIONALIZATION: EMERGING VOICES AND \\ PERSPECTIVES"
}

CONNIE JAMES

Simon Fraser University

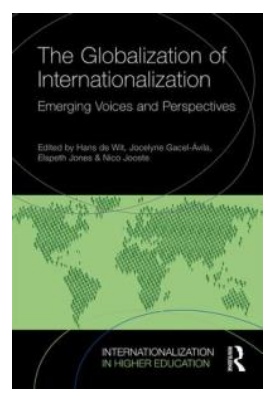

de Wit, H., Gacel-Avila, J., Jones, E., \& Jooste, N. (Eds.) (2017). The

globalization of internationalization: Emerging voices and perspectives. New York: Taylor \& Francis.

The Globalization of Internationalization edited by de Wit, Gacel-Ávila, Jones, and Jooste addresses the paucity in representation of perspectives outside the dominant Western and Anglo paradigms regarding the internationalization of higher education (HE). Accentuated at the beginning in the foreword and introduction, and then reiterated throughout the book, is the need for knowledge to move in multidirectional flows, with a de-emphasis on just knowledge dissemination from North to South. Thus, this book is an edited anthology with contributors writing from periphery locations and/or situations, all endeavoring toward a critical yet nuanced look at internationalization in a globalized world. Specifically, the book is divided into five parts: The Global Context; Politics, Conflict and Social issues; Regional Examples of Internationalization in the Emerging and Developing World; National Policies for Internationalization; and Institutional Internationalization in Emerging and Developing Contexts.

Egron-Polak's and Marmolejo's Chapter 1 serves as a signpost for the rest of the book, echoing the sentiments from the introduction but also addressing the theme behind the title, namely the "globalization OF internationalization" (p. 7, capitalization in the original). They analyze how globalization "has coloured" (p. 10) HE internationalization and its processes, as driving forces shift toward more economic rationales. The authors' discussion includes arguing for the inclusion of periphery experiences as well as analysis of contexts outside the immediate realm of HE in order to create a balanced approach with economic drivers playing a lesser role, making room for a focus on societal issues. Rizvi provides an example of contexts outside of HE 
in Chapter 2, with his look at internationalization at the schooling level. Elite schools but also even public schools across the globe are competing and internationalizing curricula, with the goal of creating future participants in a cosmopolitan global market; it is arguable that such developments can potentially influence students recruited by HEs, as well as HE pedagogy. Thus, Rizvi asserts that an examination of contexts outside of HE means understanding the different social backgrounds of students entering HE institutions, and having students critically examine their own trajectories and the global opportunities they have been afforded. Such discussions regarding social factors from this introductory section will be reflected upon throughout the rest of the volume.

Part 2 opens with an examination of refugee crises and how conflict situations should be addressed through an internationalization framework. Referencing the Syrian refugee crisis specifically, Streitweister, Miller-Idress, and de Wit argue that HE institutions can alleviate dire circumstances for many students and contribute to an overall "global stability" (p. 32) by providing access to educational institutions during times of conflict, an act that simultaneously helps displaced individuals re-adapt into society post-conflict. Chapter 4 has Heleta further examining a post-conflict context and the capacities HE institutions - in partnership with international organizations and communities - have in the rebuilding and transformation of wartorn societies. Social responsibility continues to be examined in Chapter 5 as Sánchez, Sequel and Baleisan explore a Viña del Mar University project with female entrepreneurs in rural regions of Chile. This program enabled these women to share skills with other internationals working in related fields, thereby supporting low-income groups in Chile but also lending to the joint production of "new knowledge" (p. 55). While considering the inequalities that can be produced and reproduced through education and international experiences, these scholars highlight how we can begin to have more inclusive internationalization practices.

Part 3 starts with a critical discussion of student mobility in Latin America and the Caribbean as Gacel-Ávila, Bustos-Aguirre and Freire analyze the region's mobility programs. They include recommendations to further programs and advance international and interregional interconnectivity of existing internationalization schemes. Abouchedid and Zeid then continue a look at examples of internationalization in the developing world by examining the continual rise of a more neoliberal model of privatization in the Middle East and North Africa (MENA) as this region attempts to generate income, address the growing number of individuals attending $\mathrm{HE}$, and as MENA hosts international branches of foreign universities. By contrast, Rampersad highlights publicly-funded institutions in Chapter 8, and how universities of the Englishspeaking Commonwealth are utilizing internationalization strategies and developing international relationships and ideas with other global institutions and programs to re-invest in education and HE institutions. Lastly, both Klemenčič and Taquechel extend the discussion regarding potentials for internationalization. In Chapter 9, Klemenčič further investigates the intricacies of internationalization processes in periphery locations, illustrating the importance and need for there to be deliberate strategies and an integrated and institutionally-central model within these locations, and ties and partnerships with larger society. Meanwhile, in Chapter 10, Taquechel 
introduces potential barriers to internationalization progressing in Latin America and ends Part 3 by specifically addressing issues such as the heterogeneity in institutional cultures across multiple diverse universities, as well as lack of regionalization and policies, or political and governmental commitment. He suggests prospective approaches to tackling such barriers through integration and harnessing a multidisciplinary heterogeneity as an asset.

The above scholars introduce us to issues and approaches regarding partnerships but also policies and government involvement. Part 4 delves deeper into policies vis à vis internationalization at the national level. Ustyuzhantseva begins by taking readers through a history of Russian internationalization processes as Russia transformed from state-run, to a market environment, and then returned to a non-market environment under stricter state governance and federal budgeting. He includes an analysis of the subsequent effects ensuing from this major shit and receding academic autonomy. Ziguars and Pham conduct a similar historical overview, this time of Vietnam's educational governance throughout the ages of colonial rule, Soviet influence, and leading into the present day. The scholars critically evaluate Vietnam's current national goals, which include increasing HE enrolment, global partnership, and internationalization for advancing skills and expanding overall participation in the global economy. Yang then takes us into the China context wherein, like Vietnam, the setting has been much affected by colonialism, imperialism and outside and/or Western influences. Thus, Yang instead explores a context in which internationalization has been a complex and oftentimes conflicted notion, a process fraught with tension on varying levels, from the individual to the systemic and societal. Part 4 concludes with a more hopeful presentation of internationalization. Through interview data with international students, Singh explores Malaysia as a growing student hub, looking specifically at the Malaysian government's approach of exercising soft power, and the possibilities this internationalization agenda has for developing global talent through knowledge transfer.

Part 5 - the concluding segment - continues the earlier postcolonial dialogue with Hagenmeier's look at a rural-based South African university and its emergent internationalization strategies. Hagenmeier provides rationales and suggestions toward further development, making the case for internationalization while considering debates regarding whether or not internationalizing would diminish prioritization of local and community knowledge and culture. Debates regarding internationalization are lengthened with Chapter 16's discussion of whether and how internationalization of the curriculum should progress in Kazakhstan, through analysis of interview excerpts of HE leaders and faculty sharing their opinions on four cases of internationalization initiatives and study programs. In Chapter 17 Dash also draws on interview data - this time derived from one private Cambodian university's stakeholders and their evaluation of their institution's internationalization - in order to weigh- in on the case of Cambodia. Data indicates that there is a "desire" for internationalization, particularly with certain elements of the curriculum, and calls for improvement in other dimensions. Finally, Gyamera closes this section and the main body of this book by reintroducing earlier themes and issues of Western dominance, imperialism and colonialism. 
Through a look at Ghana's public universities, Gymera critiques the practice of measuring Ghana's internationalization by Western standards, as well as the sometimes overly neoliberal and corporate underpinnings of internationalizing, all the while emphasizing the need to consider African knowledge systems and community engagement in educational processes.

The conclusion to this volume, provided by several of the editors, is not a statement of anything conclusive, but rather a summary of the themes from the book. While summarizing, de Wit, Gacel-Ávila and Jones question internationalization processes amidst a system based on commercialization, competition and a dominant Western model of operation. The scholars argue that as internationalization becomes more and more globalized, we need to weigh approaches and underlying ideologies with the integration of difference perspectives and voices; only then can we begin to gauge how to move toward "innovative and socially inclusive internationalization processes" (p. 233). Such a conclusion is in line with their intentions for this book, which was not to provide an all-encompassing answer to internationalization issues. In this sense, the editors succeed in addressing their initial goals introduced at the beginning of the book, which involve raising questions that problematize internationalization processes, while making room for periphery voices that might offer alternative responses.

Altogether, the sub topics and themes of this book, while synchronized under the broader subjects of internationalization and globalization, are quite extensive and wide-ranging. Thus, even though the book's composition can be very informative and is fitting for the editors' goal of providing alternative viewpoints, it might also not suit every reader. However, there is enough interesting scholarship in The Globalization of Internationalization that a reader should be able to find concepts pertinent to his or her context. Moreover, readers might find a new element that can lead them on a potentially enlightening path, especially if they are new to the topic of internationalization or newly delving into research on internationalization. For example, those interested in internationalization policies might be informed by literature more so in the realm of applied linguistics and how English has become synonymous with internationalization, which therefore in turn can influence policy. And just as Rizvi explains in Chapter 2 the ties between his exploration of schooling and the internationalization of $\mathrm{HE}$, there is still an overall interconnectedness of the different themes and different voices. This interconnectedness is made more apparent because of how the book is organized and presented, with its carefully selected and parsed sections. Additionally, with each chapter being relatively short, readers are given just enough information about a given topic without being overloaded, and can seek more if necessary.

More specifically, this book can be included on the syllabus for courses in Education. For instance, it can benefit graduate students as several chapters in the book are derived from empirical studies, which can potentially guide novice researchers' foray into research of internationalization. However, as there is increasingly more "attention [given] to the international dimension of higher education" (de Wit, Gacel-Ávila, \& Jones, 2017, p. 221), all students seeking to work in the field of education require a degree of understanding of major shifts in education and the influences globalized 
internationalization processes have on educational systems and policies at the international level, filtering down to the local, and vice versa. While there is a considerable amount of scholarship on internationalization, this book, which is arguably an outlier in its goals and authorship, adds a complexity that can allow students to critically examine the subject. To conclude, the Globalization of Internationalization can be valuable for anyone working in the field of education, as the book provides a level of criticality in its exploration of internationalization processes obtained from periphery sources, all the while still alluding to and leaving room for many possibilities within the realm of internationalization of $\mathrm{HE}$. 